\title{
Orthogonal Stability of Mixed Additive-Quadratic Jensen Type Functional Equation in Multi-Banach Spaces
}

\author{
Xiuzhong Yang1,2, Lidan Chang1, Guofen Liu' ${ }^{1,2}$ \\ ${ }^{1}$ College of Mathematics and Information Science, Hebei Normal University, Shijiazhuang, China \\ ${ }^{2}$ Hebei Key Laboratory of Computational Mathematics and Applications, Shijiazhuang, China \\ Email: xiuzhongyang@126.com, changlidan@yeah.net, liugf2003@163.com
}

Received 1 April 2015; accepted 30 April 2015; published 5 May 2015

Copyright @ 2015 by authors and Scientific Research Publishing Inc.

This work is licensed under the Creative Commons Attribution International License (CC BY).

http://creativecommons.org/licenses/by/4.0/

(c) (i) Open Access

\section{Abstract}

In this paper, we prove the Hyers-Ulam stability of the following mixed additive-quadratic Jensen type functional equation: $2 f\left(\frac{x+y}{2}\right)+f\left(\frac{x-y}{2}\right)+f\left(\frac{y-x}{2}\right)=f(x)+f(y)$.

\section{Keywords}

Hyers-Ulam Stability, Additive-Quadratic Jensen Type Functional Equation, Multi-Banach Spaces, Fixed Point Method

\section{Introduction}

In 1940, Ulam [1] proposed the stability problem of functional equations concerning the stability of group homomorphisms. Suppose that $\left(G_{1}, \cdot\right)$ is a group and that $\left(G_{2}, *\right)$ is a metric group with the metric $d(\cdot, \cdot)$. Given $\varepsilon>0$, does there exist a $\delta>0$ such that if a mapping $h: G_{1} \rightarrow G_{2}$ satisfies the inequality

$$
d(h(x \cdot y), h(x) * h(y))<\delta
$$

for all $x, y \in G_{1}$, then a homomorphism $H: G_{1} \rightarrow G_{2}$ exists with $d(h(x), H(x))<\varepsilon$ for all $x \in G_{1}$ ?

The case of approximately additive functions was solved by Hyers [2] under the assumption that $G_{1}$ and $G_{2}$ are Banach spaces. In 1978, Rassias [3] proved a generalization of the Hyers theorem for additive mappings. The result of Rassias has provided a lot of influences during the past more than three decades in the development of a generalization of the Hyers-Ulam stability concept. This new concept is known as Hyers-Ulam-Rassias 
stability of functional equation.

The stability problems of several functional equations have been extensively investigated by a number of authors and there are many interesting results concerning this problem. A large list of references can be found in [4]-[11].

Pinsker [12] characterized orthogonal additive functional equation on an inner product space. The orthogonal Cauchy functional equation

$$
f(x+y)=f(x)+f(y), \quad x \perp y
$$

in which $\perp$ is an orthogonality relation, is first investigated by Gudder and Strawther [13]. In 1985, Rätz [14] introduced a new definition of orthogonality by using more restrictive axioms than Gudder and Strawther. Moreover, he investigated the structure of orthogonally additive mappings. Rätz and Szabó [15] investigated the problem in a rather more general framework.

In [16], Kenary and Cho proved the Hyers-Ulam-Rassias stability of mixed additive-quadratic Jensen type functional equation in non-Archimedean normed spaces and random normed spaces. In this paper, we prove the Hyers-Ulam stability of the following mixed additive-quadratic Jensen type functional equation:

$$
2 f\left(\frac{x+y}{2}\right)+f\left(\frac{x-y}{2}\right)+f\left(\frac{y-x}{2}\right)=f(x)+f(y)
$$

in multi-Banach spaces.

The notion of multi-normed space is introduced by Dales and Polyakov [17]. This concept is somewhat similar to operator sequence space and has some connections with operator spaces and Banach lattices. Motivations for the study of multi-normed spaces and many examples are given in [17]. Also, the stability problems in multi-Banach spaces are studied by Dales and Moslehian [18], Moslehian et al. ([19]-[21]) and Wang et al. [22].

Now, let us recall some concepts concerning multi-Banach space.

Let $(E,\|\cdot\|)$ be a complex normed space, and let $k \in \mathbb{N}$. We denote by $E^{k}$ the linear space $E \oplus E \oplus \cdots \oplus E$ consisting of k-tuples $\left(x_{1}, \cdots, x_{k}\right)$, where $x_{1}, \cdots, x_{k} \in E$. The linear operations on $E^{k}$ are defined coordinate wise. The zero element of either $E$ or $E^{k}$ is denoted by 0 . We denote by $\mathbb{N}_{k}$ the set $\{1,2, \cdots, k\}$ and by $\Omega_{k}$ the group of permutations on $k$ symbols.

Definition 1.1 ([17]) A multi-norm on $\left\{E^{k}: k \in \mathbb{N}\right\}$ is a sequence

$$
\left(\|\cdot\|_{k}\right)=\left(\|\cdot\|_{k}: k \in \mathbb{N}\right)
$$

such that \|\|$_{k}$ is a norm on $E^{k}$ for each $k \in \mathbb{N},\|x\|_{1}=\|x\|$ for each $x \in E$, and the following axioms are satisfied for each $k \in \mathbb{N}$ with $k \geq 2$ :

(A1)

$$
\left\|\left(x_{\sigma(1)}, \cdots, x_{\sigma(k)}\right)\right\|_{k}=\left\|\left(x_{1}, \cdots, x_{k}\right)\right\|_{k}\left(\sigma \in \Omega_{k}, x_{1}, \cdots, x_{k} \in E\right) ;
$$

$$
\left\|\left(\alpha_{1} x_{1}, \cdots, \alpha_{k} x_{k}\right)\right\|_{k} \leq\left(\max _{i \in \mathbb{N}_{k}}\left|\alpha_{i}\right|\right)\left\|\left(x_{1}, \cdots, x_{k}\right)\right\|_{k}\left(\alpha_{1}, \cdots, \alpha_{n} \in \mathbb{C}, x_{1}, \cdots, x_{k} \in E\right) ;
$$

(A4) $\left\|\left(x_{1}, \cdots, x_{k-1}, x_{k-1}\right)\right\|_{k}=\left\|\left(x_{1}, \cdots, x_{k-1}\right)\right\|_{k-1}\left(x_{1}, \cdots, x_{k-1} \in E\right)$.

In this case, we say that $\left(\left(E^{k},\|\cdot\|_{k}\right): k \in \mathbb{N}\right)$ is a multi-normed space.

Suppose that $\left(\left(E^{k},\|\cdot\|_{k}\right): k \in \mathbb{N}\right)$ is a multi-normed space and take $k \in \mathbb{N}$. We need two properties of multi-norms which can be found in [17].

(a) $\|(x, \cdots, x)\|_{k}=\|x\| \quad(x \in E)$;

(b) $\max _{i \in \mathbb{N}_{k}}\left\|x_{i}\right\| \leq\left\|\left(x_{1}, \cdots, x_{k}\right)\right\|_{k} \leq \sum_{i=1}^{k}\left\|x_{i}\right\| \leq k \max _{i \in \mathbb{N}_{k}}\left\|x_{i}\right\| \quad\left(x_{1}, \cdots, x_{k} \in E\right)$.

It follows from (b) that, if $(E,\|\cdot\|)$ is a Banach space, then $\left(E^{k},\|\cdot\|_{k}\right)$ is a Banach space for each $k \in \mathbb{N}$; in this case, $\left(\left(E^{k},\|\cdot\|_{k}\right): k \in \mathbb{N}\right)$ is a multi-Banach space. 
Now, we state two important examples of multi-norms for an arbitrary normed space $E$ (see, for details, [17]). Example 1.2 ([17]) The sequence $\left(\|\|_{k}: k \in \mathbb{N}\right)$ on $\left\{E^{k}: k \in \mathbb{N}\right\}$ defined by

$$
\left\|x_{1}, \cdots, x_{k}\right\|_{k}:=\max _{i \in \mathbb{N}_{k}}\left\|x_{i}\right\|, \quad\left(x_{1}, \cdots, x_{k} \in E\right)
$$

is a multi-norm called the minimum multi-norm. The terminology "minimum" is justified by property (b).

Example 1.3 ([17]) Let $\left\{\left(\|\|_{k}: k \in \mathbb{N}\right): \alpha \in A\right\}$ be the (non-empty) family of all multi-norms on $\left\{E^{k}: k \in \mathbb{N}\right\}$. For $k \in \mathbb{N}$, set

$$
\left\|x_{1}, \cdots, x_{k}\right\|_{k}:=\sup _{\alpha \in A}\left\|x_{1}, \cdots, x_{k}\right\|_{k}^{\alpha}, \quad\left(x_{1}, \cdots, x_{k} \in E\right) .
$$

Then $\left(\|\cdot\|_{k}: k \in \mathbb{N}\right)$ is a multi-norm on $\left\{E^{k}: k \in \mathbb{N}\right\}$, which is called the maximum multi-norm.

We need the following observation which can be easily deduced from the triangle inequality for the norm $\|\cdot\|_{k}$ and the property (b) of multi-norms.

Lemma 1.4 [17] Suppose that $k \in \mathbb{N}$ and $\left(x_{1}, \cdots, x_{k}\right) \in E^{k}$. For each $j \in\{1, \cdots, k\}$, let $\left\{x_{n}^{j}\right\}_{n \in \mathbb{N}}$ be a sequence in $E$ such that $\lim _{n \rightarrow \infty} x_{n}^{j}=x_{j}$. Then for each $\left(y_{1}, \cdots, y_{k}\right) \in E^{k}$, we have

$$
\lim _{n \rightarrow \infty}\left(x_{n}^{1}-y_{1}, \cdots, x_{n}^{k}-y_{k}\right)=\left(x_{1}-y_{1}, \cdots, x_{k}-y_{k}\right) \text {. }
$$

Definition 1.5 [17] Let $\left(\left(E^{k},\|\cdot\|_{k}\right): k \in \mathbb{N}\right)$ be a multi-normed space. A sequence $\left\{x_{n}\right\}$ in $E$ is a multi-null sequence if, for each $\varepsilon>0$, there exists $n_{0} \in \mathbb{N}$ such that

$$
\sup _{k \in \mathbb{N}}\left\|x_{n}, \cdots, x_{n+k-1}\right\|_{k}<\varepsilon, \quad\left(n \geq n_{0}\right) .
$$

Let $x \in E$. We say that the sequence $\left\{x_{n}\right\}$ is multi-convergent to $x$ in $E$ and write

$$
\lim _{n \rightarrow \infty} x_{n}=x .
$$

if $\left\{x_{n}-x\right\}$ is a multi-null sequence.

There are several orthogonality notations on a real normed space available. But here, we present the orthogonal concept introduced by Rätz [14]. This is given in the following definition.

Definition 1.6 Suppose that $X$ is a vector space (algebraic module) with $\operatorname{dim} X \geq 2$, and $\perp$ is a binary relation on $X$ with the following properties:

1) Totality of $\perp$ for zero: $x \perp 0,0 \perp x$ for all $x \in X$;

2) Independence: if $x, y \in X-\{0\}$ and $x \perp y$, then $x$ and $y$ are linearly independent;

3) Homogeneity: if $x, y \in X$ and $x \perp y$, then $\alpha x \perp \beta y$ for all $\alpha, \beta \in \mathbb{R}$;

4) Thalesian properity: if $P$ is a 2-dimensional subspace of $X, \quad x \in P$ and $\lambda \in \mathbb{R}_{+}$, which is the set of nonnegative real numbers, then there exists $y_{0} \in P$ such that $x \perp y_{0}$ and $x+y_{0} \perp \lambda x-y_{0}$.

The pair $(X, \perp)$ is called an orthogonality space (resp., module). By an orthogonality normed space (normed module) we mean an orthogonality space (resp., module) having a normed (resp., normed module) structure.

Definition 1.7 Let $X$ be a set. A function $d: X \times X \rightarrow[0, \infty]$ is called a generalized metric on $X$ if and only if $d$ satisfies

(M1) $d(x, y)=0$ if and only if $x=y$;

(M2) $d(x, y)=d(y, x)$ for all $x, y \in X$;

(M3) $d(x, z) \leq d(x, y)+d(y, z)$ for all $x, y, z \in X$.

Theorem 1.8 ([23]) Let $(X, d)$ be a generalized complete metric space. Assume that $J: X \rightarrow X$ be a strictly contractive mapping with Lipschitz constant $L<1$. Then, for all $x \in X$, either

$$
d\left(J^{n} x, J^{n+1} x\right)=\infty
$$

for all nonnegative integers $n$ or there exists a positive integer $n_{0}$ such that

1) $d\left(J^{n} x, J^{n+1} x\right)<\infty$ for all $n \geq n_{0}$;

2) the sequence $\left\{J^{n} x\right\}$ converges to a fixed point $x^{*}$ of $J$; 
3) $x^{*}$ is the unique fixed point of $J$ in the set $X^{*}=\left\{y \in X \mid d\left(J^{n_{0}} x, y\right)<\infty\right\}$;

4) $d\left(y, x^{*}\right) \leq \frac{1}{1-L} d(J y, y)$ for all $y \in X^{*}$.

\section{Hyers-Ulam Stability of Mixed Additive-Quadratic Jensen Type Functional Equation}

Throughout this section, let $\alpha>0, E$ be an orthogonality space and let $\left(\left(F^{n},\|\cdot\|_{n}\right): n \in \mathbb{N}\right)$ be a multi-Banach space. For convenience, we use the following abbreviation for a given mapping $f: E \rightarrow F$,

$$
D f(x, y)=2 f\left(\frac{x+y}{2}\right)+f\left(\frac{x-y}{2}\right)+f\left(\frac{y-x}{2}\right)-f(x)-f(y)
$$

for all $x, y \in E$ with $x \perp y$.

\subsection{Hyers-Ulam Stability of Functional Equation (1): An Odd Case}

In this section, using direct method, we prove the Hyers-Ulam stability of the functional Equation (1) in multiBanach space.

Definition 2.1 An odd mapping $f: E \rightarrow F$ is called an orthogonally Jensen additive mapping if

$$
2 f\left(\frac{x+y}{2}\right)+f\left(\frac{x-y}{2}\right)+f\left(\frac{y-x}{2}\right)=f(x)+f(y)
$$

for all $x, y \in E$ with $x \perp y$.

Theorem 2.2 Suppose that $\alpha$ is a nonnegative real number and $f_{a}: E \rightarrow F$ is an odd mapping satisfying

$$
\sup _{k \in \mathbb{N}}\left\|\left(D f_{a}\left(x_{1}, y_{1}\right), \cdots, D f_{a}\left(x_{k}, y_{k}\right)\right)\right\|_{k} \leq \alpha
$$

for all $x_{1}, \cdots, x_{k}, y_{1}, \cdots, y_{k} \in E$ and $x_{i} \perp y_{i}(i=1, \cdots, k)$. Then there exists a unique orthogonally Jensen additive mapping $A: E \rightarrow F$ such that

$$
\sup _{k \in \mathbb{N}}\left\|\left(f_{a}\left(x_{1}\right)-A\left(x_{1}\right), \cdots, f_{a}\left(x_{k}\right)-A\left(x_{k}\right)\right)\right\|_{k} \leq \alpha
$$

for all $x_{1}, \cdots, x_{k} \in E$.

Proof. Replacing $y_{1}, \cdots, y_{k}$ by $0, \cdots, 0$ in (2.1), we get

$$
\sup _{k \in \mathbb{N}}\left\|\left(2 f_{a}\left(\frac{x_{1}}{2}\right)-f_{a}\left(x_{1}\right), \cdots, 2 f_{a}\left(\frac{x_{k}}{2}\right)-f_{a}\left(x_{k}\right)\right)\right\|_{k} \leq \alpha
$$

for all $x_{1}, \cdots, x_{k} \in E$ since $0 \perp x_{i}(i=1, \cdots, k)$. Replacing $x_{1}, \cdots, x_{k}$ by $2^{n} x_{1}, \cdots, 2^{n} x_{k}$ in (2.3) and dividing both sides by $2^{n}$, we get

$$
\sup _{k \in \mathbb{N}}\left\|\left(\frac{f_{a}\left(2^{n-1} x_{1}\right)}{2^{n-1}}-\frac{f_{a}\left(2^{n} x_{1}\right)}{2^{n}}, \cdots, \frac{f_{a}\left(2^{n-1} x_{k}\right)}{2^{n-1}}-\frac{f_{a}\left(2^{n} x_{k}\right)}{2^{n}}\right)\right\|_{k} \leq 2^{-n} \alpha
$$

for all $x_{1}, \cdots, x_{k} \in E$ since $0 \perp 2^{n} x_{i}(i=1, \cdots, k)$. By using (2.4) and the principle of mathematical induction, we can easily get

$$
\sup _{k \in \mathbb{N}}\left\|\left(\frac{f_{a}\left(2^{n+m} x_{1}\right)}{2^{n+m}}-\frac{f_{a}\left(2^{n} x_{1}\right)}{2^{n}}, \cdots, \frac{f_{a}\left(2^{n+m} x_{k}\right)}{2^{n+m}}-\frac{f_{a}\left(2^{n} x_{k}\right)}{2^{n}}\right)\right\|_{k} \leq \alpha \sum_{i=n+1}^{n+m} 2^{-i}
$$

for all $x_{1}, \cdots, x_{k} \in E, n, m \in \mathbb{N}, m \geq 1$.

We now fix $x \in E$. We have 


$$
\begin{aligned}
& \sup _{k \in \mathbb{N}}\left\|\left(\frac{f_{a}\left(2^{n+m} x\right)}{2^{n+m}}-\frac{f_{a}\left(2^{n} x\right)}{2^{n}}, \cdots, \frac{f_{a}\left(2^{n+m+k-1} x\right)}{2^{n+m+k-1}}-\frac{f_{a}\left(2^{n+k-1} x\right)}{2^{n+k-1}}\right)\right\|_{k} \\
& =\sup _{k \in \mathbb{N}} \|\left(\frac{f_{a}\left(2^{n+m} x\right)}{2^{n+m}}-\frac{f_{a}\left(2^{n} x\right)}{2^{n}}, \cdots, \frac{1}{2^{k-1}}\left(\frac{f_{a}\left(2^{n+m}\left(2^{k-1} x\right)\right)}{2^{n+m}}-\frac{f_{a}\left(2^{n}\left(2^{k-1} x\right)\right)}{2^{n}}\right) \|_{k}\right. \\
& \quad \leq\left\|\left(\frac{f_{a}\left(2^{n+m} x\right)}{2^{n+m}}-\frac{f_{a}\left(2^{n} x\right)}{2^{n}}, \cdots, \frac{f_{a}\left(2^{n+m}\left(2^{k-1} x\right)\right)}{2^{n+m}}-\frac{f_{a}\left(2^{n}\left(2^{k-1} x\right)\right)}{2^{n}}\right)\right\|_{k} \\
& \leq \alpha \sum_{i=n+1}^{n+m} 2^{-i} .
\end{aligned}
$$

where we have used the Definition 1.1 and also replaced $x_{1}, \cdots, x_{k}$ by $x, 2 x, \cdots, 2^{k-1} x$ in (2.5). It follows that $\left\{\frac{f_{a}\left(2^{n} x\right)}{2^{n}}\right\}$ is a Cauchy sequence and so it is convergent in the multi-Banach spaces $F$. Set

$$
A(x)=\lim _{n \rightarrow \infty} \frac{f_{a}\left(2^{n} x\right)}{2^{n}}
$$

for all $x \in E$. Hence, for each $\varepsilon>0$, there exists $n_{0}$ such that

$$
\sup _{k \in \mathbb{N}}\left\|\left(\frac{f_{a}\left(2^{n} x\right)}{2^{n}}-A(x), \cdots, \frac{f_{a}\left(2^{n+k-1} x\right)}{2^{n+k-1}}-A(x)\right)\right\|_{k}<\varepsilon
$$

for all $n \geq n_{0}$. In particular, by property (b) of multi-norms, we have

$$
\lim _{n \rightarrow \infty}\left\|\frac{f_{a}\left(2^{n} x\right)}{2^{n}}-A(x)\right\|=0, \quad(x \in E) .
$$

We next put $n=0$ in (2.5) to get

$$
\sup _{k \in \mathbb{N}}\left\|\left(\frac{f_{a}\left(2^{m} x\right)}{2^{m}}-f_{a}\left(x_{1}\right), \cdots, \frac{f_{a}\left(2^{m} x\right)}{2^{m}}-f_{a}\left(x_{k}\right)\right)\right\|_{k} \leq \alpha \sum_{i=1}^{m} 2^{-i} .
$$

Letting $m \rightarrow \infty$ and using Lemma 1.4 and (2.6), we obtain

$$
\sup _{k \in \mathbb{N}}\left\|\left(A\left(x_{1}\right)-f_{a}\left(x_{1}\right), \cdots, A\left(x_{k}\right)-f_{a}\left(x_{k}\right)\right)\right\|_{k} \leq \alpha .
$$

Let $x, y \in E$ and $x \perp y$. Considering Definition 1.6, we have $2^{n} x \perp 2^{n} y$. Put $x_{1}=\cdots=x_{k}=2^{n} x$, $y_{1}=\cdots=y_{k}=2^{n} y$ in (2.1) and divide both sides by $2^{n}$. Then, using property (a) of multi-norms, we obtain

$$
\left\|\frac{f_{a}\left(2^{n} \cdot \frac{x+y}{2}\right)}{2^{n-1}}+\frac{f_{a}\left(2^{n} \cdot \frac{x-y}{2}\right)}{2^{n}}+\frac{f_{a}\left(2^{n} \cdot \frac{y-x}{2}\right)}{2^{n}}-\frac{f_{a}\left(2^{n} x\right)}{2^{n}}-\frac{f_{a}\left(2^{n} y\right)}{2^{n}}\right\| \leq 2^{-n} \alpha
$$

for all $x, y \in E$ and $x \perp y$. Taking $n \rightarrow \infty$, we get

$$
2 A\left(\frac{x+y}{2}\right)+A\left(\frac{x-y}{2}\right)+A\left(\frac{y-x}{2}\right)-A(x)-A(y)=0
$$


for all $x, y \in E$ and $x \perp y$. Since $f$ is an odd mapping, according to the definition of $A$, we know that $A$ is an odd mapping. By Definition 2.1, the mapping $A$ is an orthogonally additive mapping.

If $A^{\prime}$ is another orthogonally additive mapping satisfying (2.2), then

$$
\begin{aligned}
\left\|A(x)-A^{\prime}(x)\right\| & =\frac{1}{2^{n}}\left\|A\left(2^{n} x\right)-A^{\prime}\left(2^{n} x\right)\right\| \\
& \leq \frac{1}{2^{n}}\left\|A\left(2^{n} x\right)-f_{a}\left(2^{n} x\right)\right\|+\frac{1}{2^{n}}\left\|f_{a}\left(2^{n} x\right)-A^{\prime}\left(2^{n} x\right)\right\| \\
& \leq \frac{1}{2^{n}} \cdot 2 \alpha .
\end{aligned}
$$

Taking $n \rightarrow \infty$, we get $A=A^{\prime}$. This completes the proof.

\subsection{Hyers-Ulam Stability of Functional Equation (1): An Even Case}

In this section, we prove the Hyers-Ulam stability of the functional Equation (1) in multi-Banach space with the fixed point method.

Definition 2.3 An even mapping $f: E \rightarrow F$ is called an orthogonally Jensen quadratic mapping if

$$
2 f\left(\frac{x+y}{2}\right)+f\left(\frac{x-y}{2}\right)+f\left(\frac{y-x}{2}\right)=f(x)+f(y)
$$

for all $x, y \in E$ with $x \perp y$.

Theorem 2.4 Suppose that $\alpha$ is a nonnegative real number and $f_{q}: E \rightarrow F$ is an even mapping satisfying

$$
\sup _{k \in \mathbb{N}}\left\|\left(D f_{q}\left(x_{1}, y_{1}\right), \cdots, D f_{q}\left(x_{k}, y_{k}\right)\right)\right\|_{k} \leq \alpha
$$

for all $x_{1}, \cdots, x_{k}, y_{1}, \cdots, y_{k} \in E$ and $x_{i} \perp y_{i}(i=1, \cdots, k)$ and $f_{q}(0)=0$. Then there exists a unique orthogonally Jensen quadratic mapping $Q: E \rightarrow F$ such that

$$
\sup _{k \in \mathbb{N}}\left\|\left(f_{q}\left(x_{1}\right)-Q\left(x_{1}\right), \cdots, f_{q}\left(x_{k}\right)-Q\left(x_{k}\right)\right)\right\|_{k} \leq \frac{1}{3} \alpha
$$

for all $x_{1}, \cdots, x_{k} \in E$.

Proof. Letting $y_{1}=y_{2}=\cdots=y_{k}=0$ in (2.7), we get

$$
\sup _{k \in \mathbb{N}}\left\|\left(4 f_{q}\left(\frac{x_{1}}{2}\right)-f_{q}\left(x_{1}\right), \cdots, 4 f_{q}\left(\frac{x_{k}}{2}\right)-f_{q}\left(x_{k}\right)\right)\right\|_{k} \leq \alpha
$$

for all $x_{1}, \cdots, x_{k} \in E$ since $0 \perp x_{i}(i=1, \cdots, k)$. Replacing $\frac{x_{1}}{2}, \frac{x_{2}}{2}, \cdots, \frac{x_{k}}{2}$ by $x_{1}, \cdots, x_{k}$ and dividing both sides by 4 , we get

$$
\sup _{k \in \mathbb{N}}\left\|\left(\frac{1}{4} f_{q}\left(2 x_{1}\right)-f_{q}\left(x_{1}\right), \cdots, \frac{1}{4} f_{q}\left(2 x_{k}\right)-f_{q}\left(x_{k}\right)\right)\right\|_{k} \leq \frac{1}{4} \alpha
$$

Let $S=\{g: X \rightarrow Y \mid g(0)=0\}$ and introduce the generalized metric $d$ defined on $S$ by

$$
d(g, h)=\inf \left\{c \in[0, \infty] \mid \sup _{k \in \mathbb{N}}\left\|\left(g\left(x_{1}\right)-h\left(x_{1}\right), \cdots, g\left(x_{k}\right)-h\left(x_{k}\right)\right)\right\|_{k} \leq c \text {, for } x_{1}, \cdots, x_{k} \in E\right\}
$$

Then it is easy to show that $(S, d)$ is a generalized complete metric space (see [5], Lemma 2.1).

We now define an operator $J: E \rightarrow E$ by

$$
J g(x)=\frac{1}{4} g(2 x), \quad \forall x \in E .
$$


we assert that $J$ is a strictly contractive operator. Given $g, h \in S$, let $c \in[0, \infty]$ be an arbitrary constant with $d(g, h) \leq c$. From the definition of $d$, it follows that

$$
\sup _{k \in \mathbb{N}}\left\|\left(g\left(x_{1}\right)-h\left(x_{1}\right), \cdots, g\left(x_{k}\right)-h\left(x_{k}\right)\right)\right\|_{k} \leq c
$$

for all $x_{1}, \cdots, x_{k} \in E$. Therefore

$$
\left\|\left(J g\left(x_{1}\right)-J h\left(x_{1}\right), \cdots, J g\left(x_{k}\right)-J h\left(x_{k}\right)\right)\right\|_{k}=\left\|\left(\frac{1}{4} g\left(2 x_{1}\right)-\frac{1}{4} h\left(2 x_{1}\right), \cdots, \frac{1}{4} g\left(2 x_{k}\right)-\frac{1}{4} h\left(2 x_{k}\right)\right)\right\|_{k} \leq \frac{1}{4} c
$$

for all $x_{1}, \cdots, x_{k} \in E$. Hence, it holds that $d(J g, J h) \leq \frac{1}{4} c$, i.e., $d(J g, J h) \leq \frac{1}{4} d(g, h)$ for all $g, h \in S$. This means that $J$ is a strictly contractive operator on $S$ with the Lipschitz constant $L=\frac{1}{4}$.

By (2.10), we have $d\left(J f_{q}, f_{q}\right) \leq \frac{1}{4} \alpha<\infty$. According to Theorem 1.8, we deduce the existence of a fixed point of $J$, that is, the existence of a mapping $Q: X \rightarrow Y$ such that $Q(2 x)=4 Q(x)$ for all $x \in E$. Moreover, we have $d\left(J^{n} f_{q}, Q\right) \rightarrow 0$, which implies

$$
Q(x)=\lim _{n \rightarrow \infty} J^{n} f_{q}(x)=\lim _{n \rightarrow \infty} \frac{f_{q}\left(2^{n} x\right)}{4^{n}}
$$

for all $x \in E$. Also, $d\left(f_{q}, Q\right) \leq \frac{1}{1-L} d\left(J f_{q}, f_{q}\right)$ implies the inequality

$$
d\left(f_{q}, Q\right) \leq \frac{1}{1-\frac{1}{4}} d\left(J f_{q}, f_{q}\right) \leq \frac{1}{3} \alpha .
$$

Let $x, y \in E$ and $x \perp y$. Considering Definition 1.6, we have $2^{n} x \perp 2^{n} y$. Set $x_{1}=\cdots=x_{k}=2^{n} x$, $y_{1}=\cdots=y_{k}=2^{n} y$ in (2.7) and divide both sides by $4^{n}$. Then, using property (a) of multi-norms, we obtain

$$
\| \frac{2 f_{a}\left(2^{n} \cdot \frac{x+y}{2}\right)}{4^{n}}+\frac{f_{a}\left(2^{n} \cdot \frac{x-y}{2}\right)}{4^{n}}+\frac{f_{a}\left(2^{n} \cdot \frac{y-x}{2}\right)}{4^{n}}-\frac{f_{a}\left(2^{n} x\right)}{4^{n}}-\frac{f_{a}\left(2^{n} y\right)}{4^{n}} \mid \leq \frac{\alpha}{4^{n}}
$$

for all $x, y \in E$ and $x \perp y$. Taking $n \rightarrow \infty$, we get

$$
2 Q\left(\frac{x+y}{2}\right)+Q\left(\frac{x-y}{2}\right)+Q\left(\frac{y-x}{2}\right)-Q(x)-Q(y)=0
$$

for all $x, y \in E$ and $x \perp y$. Since $f$ is an even mapping, $Q$ is an even mapping. According to Definition 2.3, we know that $Q$ is an orthogonally quadratic mapping.

The uniqueness of $Q$ follows from the fact that $Q$ is the unique fixed point of $J$ with the property that there exists $l \in(0, \infty)$ such that

$$
\sup _{k \in \mathbb{N}}\left\|\left(f_{q}\left(x_{1}\right)-Q\left(x_{1}\right), \cdots, f_{q}\left(x_{k}\right)-Q\left(x_{k}\right)\right)\right\|_{k} \leq l
$$

for all $x_{1}, \cdots, x_{k} \in E$. This completes the proof of the theorem.

\section{Acknowledgements}

We thank the editor and the referee for their comments. Research is funded by the National Natural Science 
Foundation of China grant 11371119 and by Natural Science Foundation of Education Department of Hebei Province grant Z2014031.

\section{References}

[1] Ulam, S.M. (1960) A Collection of the Mathematical Problems. Inderscience Publishers, New York.

[2] Hyers, D.H. (1941) On the Stability of the Linear Functional Equation. Proceedings of the National Academy of Sciences, 27, 222-224. http://dx.doi.org/10.1073/pnas.27.4.222

[3] Rassias, Th.M. (1978) On the Stability of the Linear Mapping in Banach Spaces. Proceedings of the American Mathematical Society, 72, 297-300. http://dx.doi.org/10.1090/S0002-9939-1978-0507327-1

[4] Jung, S.M. (2011) Hyers-Ulam-Rassias Stability of Functional Equations in Nonlinear Analysis. Springer, New York. http://dx.doi.org/10.1007/978-1-4419-9637-4

[5] Mihet, D. and Radu, V. (2008) On the Stability of the Additive Cauchy Functional Equation in Random Normed Spaces. Journal of Mathematical Analysis and Applications, 343, 567-572. http://dx.doi.org/10.1016/j.jmaa.2008.01.100

[6] Zhao, X.P., Yang, X.Z. and Pang, C.T. (2013) Solution and Stability of a General Mixed Type Cubic and Quartic Functional Equation. Journal of Function Spaces and Applications, 2013, Article ID: 673810.

[7] Moslehian, M.S. and Rassias, Th.M. (2007) Orthogonal Stability of Additive Type Equations. Aequationes Mathematicae, 73, 249-259. http://dx.doi.org/10.1007/s00010-006-2868-0

[8] Najati, A. (2008) On the Stability of a Quartic Functional Equation. Journal of Mathematical Analysis and Applications, 340, 569-574. http://dx.doi.org/10.1016/j.jmaa.2007.08.048

[9] Park, C., Cho, Y. and Kenary, H.A. (2012) Orthogonal Stability of a Generalized Quadratic Functional Equation in Non-Archimedean Spaces. Journal of Mathematical Analysis and Applications, 14, 526-535.

[10] Yang, X., Chang, L., Liu, G. and Shen, G. (2015) Stability of Functional Equations in ( $($, $\beta$ )-Normed Spaces. Journal of Inequalities and Applications, 2015, 112. http://dx.doi.org/10.1186/s13660-015-0628-1

[11] Saadati, R. and Park, C. (2010) Non-Archimedean L-Fuzzy Normed Spaces and Stability of Functional Equations. Computers Mathematics with Applications, 60, 2488-2496. http://dx.doi.org/10.1016/j.camwa.2010.08.055

[12] Pinsker, A.G. (1938) Sur une fonctionnelle dans l'espace de Hilbert. Comptes Rendus (Dokl.) de l'Académie des Sciences, URSS, 20, 411-414.

[13] Gudder, S. and Strawther, D. (1975) Orthogonally Additive and Orthogonally Increasing Functions on Vector Spaces. Pacific Journal of Mathematics, 58, 427-436. http://dx.doi.org/10.2140/pjm.1975.58.427

[14] Rätz, J. (1985) On Orthogonally Additive Mappings. Aequationes Mathematicae, 28, 35-49. http://dx.doi.org/10.1007/BF02189390

[15] Rätz, J. and Szabó, G. (1989) On Orthogonally Additive Mappings IV. Aequationes Mathematicae, 38, 73-85. http://dx.doi.org/10.1007/BF01839496

[16] Kenary, H.A. and Cho, Y. (2011) Stability of Mixed Additive-Quadratic Jensen Type Functional Equation in Various Spaces. Computers Mathematics with Applications, 61, 2704-2724. http://dx.doi.org/10.1016/j.camwa.2011.03.024

[17] Dales, H.G. and Moslehian, M.S. (Preprint) Multi-Normed Spaces and Multi-Banach Algebras.

[18] Dales, H.G. and Moslehian, M.S. (2007) Stability of Mappings on Multi-Normed Spaces. Glasgow Mathematical Journal, 49, 321-332. http://dx.doi.org/10.1017/S0017089507003552

[19] Moslehian, M.S. (2008) Superstability of Higher Derivations in Multi-Banach Algebras. Tamsui Oxford Journal of Information and Mathematical Sciences, 24, 417-427.

[20] Moslehian, M.S., Nikodem, K. and Popa, D. (2009) Asymptotic Aspect of the Quadratic Functional Equation on MultiNormed Spaces. Journal of Mathematical Analysis and Applications, 355, 717-724. http://dx.doi.org/10.1016/j.jmaa.2009.02.017

[21] Moslehian, M.S. and Srivastava, H.M. (2010) Jensen’s Functional Equation in Multi-Normed Spaces. Taiwanese Journal of Mathematics, 14, 453-462.

[22] Wang, L., Liu, B. and Bai, R. (2010) Stability of a Mixed Type Functional Equation on Multi-Banach Spaces: A Fixed Point Approach. Fixed Point Theory and Application, 2010, Article ID: 283827, 9 p.

[23] Diaz, J.B. and Margolis, B. (1968) A Fixed Point Theorem of the Alternative for Contractions on Generalized Complete Metric Space. Bulletin of the American Mathematical Society, 74, 305-309. http://dx.doi.org/10.1090/S0002-9904-1968-11933-0 